\title{
Metatarsophalangeal Joint Fusion using Plate a Lag Screw-Single Surgeon Experience in a District General Hospital
}

\section{Muhammad Murtaza Khan ${ }^{*}$ and Dakshinamurthy Sunderamoorthy}

Northern Lincolnshire and Goole Foundation Trust, United Kingdom

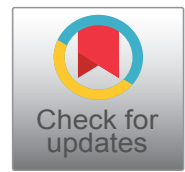

*Corresponding author: Muhammad Murtaza Khan, Northern Lincolnshire and Goole and Foundation Trust, 6 Saxby House, Church lane, Scunthorpe DN15 7HY, United Kingdom

\begin{abstract}
Aims and objectives: The aim of this study was to determine the long-term outcome of patients after MTPJ fusion of big toe using plate and lag screw system.

Material and method: We retrospectively evaluated the patients who were operated for MTPJ fusion of big toe from January 2015 to December 2018. Rate of non-union and functional outcome (pre and post op MOXFQ score) were considered as a primary outcome. Overall patient satisfaction was considered as the secondary outcome. A total of 28 patients were operated and they were followed using clinical notes. Our mean follow up was for a period of 16 months (1.34 years).
\end{abstract}

Results: The average age of our patients was 68.35 years, 2 male, 26 females, 6 with bilateral disease, 10 had right sided \& 6 had left sided disease. The use of plate with a lag screw fixation $(1 / 28)$ result in non-union rate of $3.57 \%$. Following surgery, the mean MOXFQ score was reduced from $(62.68 \pm 7.36)$ to $(21.43 \pm 12.87)$ for pre-op and post op calculations respectively with a $\mathrm{Cl}$ of $95 \%$ p value $<0.0001$ and $t$ statistic of -28.16 calculated using student $t$ test.

Conclusion: Our study demonstrated that plate with a lag screw provides best outcome with overall satisfaction of $89 \%$ after a mean follow up period of 1.34 year.

\section{Keywords}

MTPJ fusion, Plate with lag screw, MOXFQ

\section{Introduction}

The modality of choice for advanced disease involving the $\mathrm{I}^{\text {st }}$ Metatarsophalangeal joint of foot is surgical fusion of the joint [1]. The common underlying pathological conditions leading to diseased/symptomatic joint are osteoarthritis, rheumatoid arthritis, hallux rigi- dus, hallux valgus and hallux varus [2,3]. The fusion of diseased joint is considered as a reliable treatment option or In fact gold standard both in terms of restoration of function and correction of deformity [2,4].

There is no universally acceptable method of fixation. Some have agreed upon simple $\mathrm{K}$ wire fixation while others prefer more complex fixation methods like a plate with or without screw fixation or the use of external fixator as a first line option. However, other group of surgeons prefers the use of stiemen pin with cross $\mathrm{K}$ wires as implant of choice [2].

Some surgeons have proposed percutaneous fixation of joint as simple and quick procedure with a nonunion rate of $10 \%$ [5]. However, despite of all this the reported rate of non-union has been documented between 8-14\% [6].

Considering plate fixation as an implant of choice researchers are of the view that locking/non locking plate has implication regrading the stability of construct with former being less susceptible to displacement [1]. However, others are of the view that dorsal plate with compression screw is the most stable construct [7].

Moreover, the post-operative course has also been scrutinized in this modern world of evidence-based practice by the fact that approach has gradually shifted from a more orthodox way of post-operative course of non-weight bearing for a significant period with associated complications of immobility to immediate weight bearing using surgical shoes. This change of practice has again demonstrated acceptable fusion rates $[2,4]$.

Citation: Khan MM, Sunderamoorthy D (2020) Metatarsophalangeal Joint Fusion using Plate a Lag Screw-Single Surgeon Experience in a District General Hospital. Int J Foot Ankle 4:044. doi.org/10.23937/26433885/1710044

Accepted: May 04, 2020: Published: May 06, 2020

Copyright: (c) 2020 Khan MM, et al. This is an open-access article distributed under the terms of the Creative Commons Attribution License, which permits unrestricted use, distribution, and reproduction in any medium, provided the original author and source are credited. 
Considering this background in mind we evaluated the results MTPJ fusion of big toe in our institute to as certain the best implant, functional outcome and overall satisfaction following the procedure.

\section{Material and Method}

We retrospectively evaluated our patients who were operated for fusion of metatarsophalangeal joint of big toe from January 2015 to December 2018. The project was registered as a retrospective audit and was approved by the audit team of the hospital from ethical point of view. Considering this being a retrospective analysis this did not require a formal written informed consent before proceeding any further.

A total of 28 patients were identified during the said period (Figure 1). Any patient undergoing revision surgery following initial failure of fusion was excluded from the study.

A total of 28 patient met the inclusion criteria and were reviewed using clinical letters, operative notes and $\mathrm{x}$ ray/CT scan reports and films for data collection to ascertain outcome following intervention.

The primary outcome was to evaluate the rate of non-union and functional outcome in terms of pre and post-operative Manchester Oxford Foot and Ankle Questionnaire (MOXFQ score).The overall satisfaction for procedure was considered as a secondary outcome. We followed our patient for an average period of 1.3 years.

\section{Patient Demographics:}

80

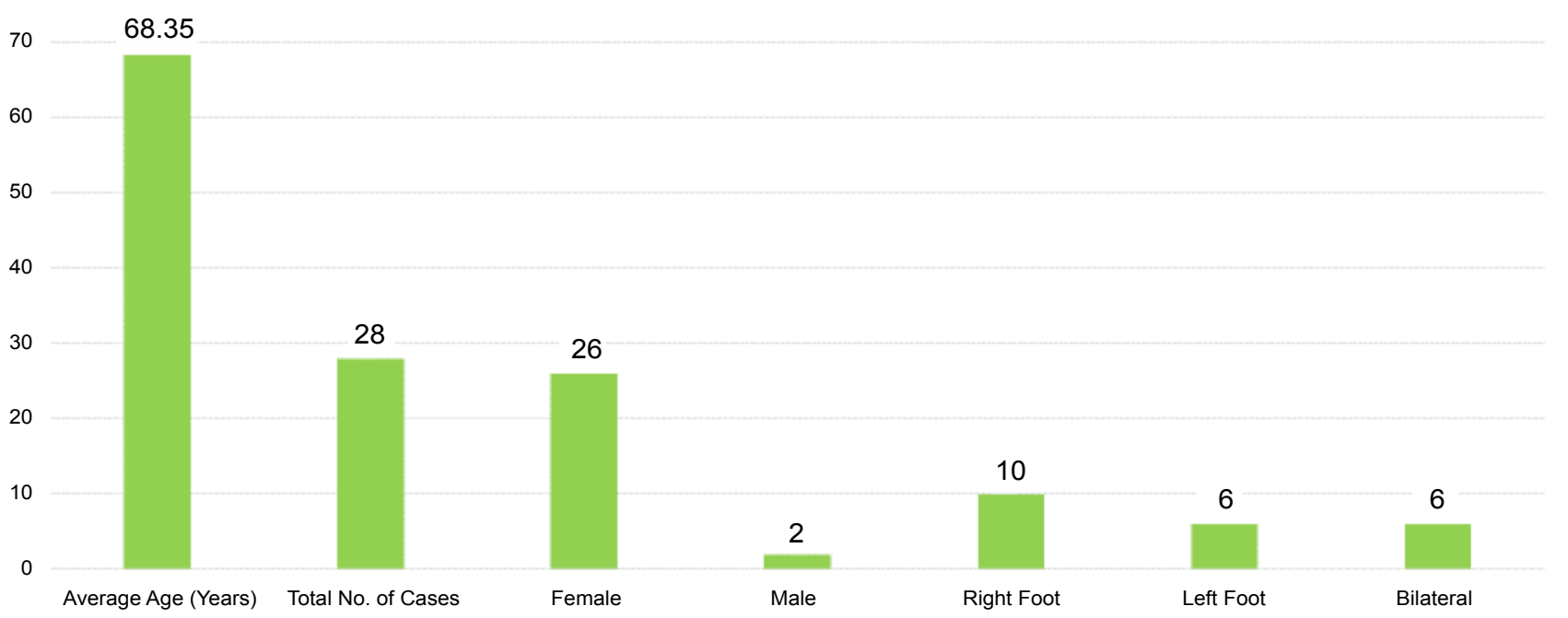

Figure 1: Patient demographics.

\section{Underlying Diagnosis}

10

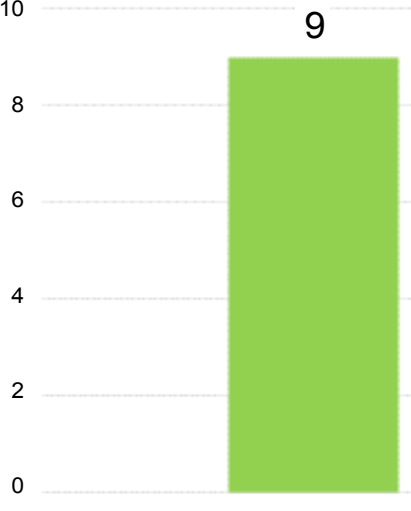

Hallux Valgus

11

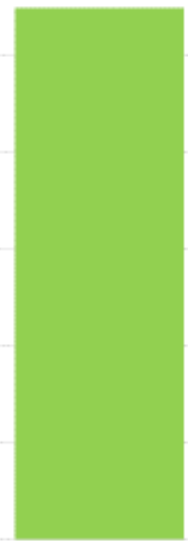

Osteoarthritis
8

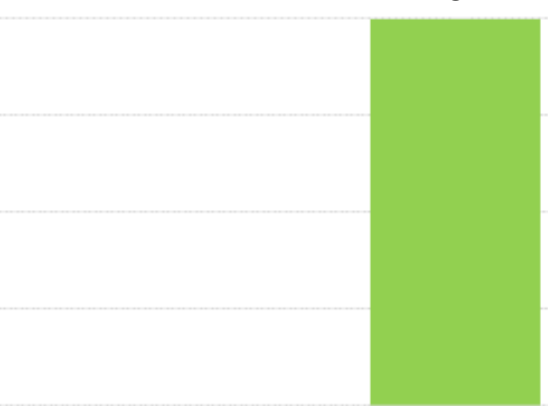

Rheumatoid Arthritis

Figure 2: Underlying diagnosis. 


\section{Results}

A total of 28 patients were evaluated, average age of our patients was 68.35 years, 2 males, 26 females, 6 with bilateral disease, 10 had right sided $\& 6$ had problems with the left foot (Figure 1). The use of plate with a lag screw fixation $(1 / 28)$ result in non-union rate of $3.57 \%$. Considering the underlying etiology of disease (Figure 2), 9 had hallux valgus, 11 had osteoarthritis and 8 suffer from rheumatoid arthritis.

$1 / 28(3.57 \%)$ suffered from non-union when plate with lag screw was used (Figure 3). The underlying etiology in this isolated case of non-union was osteoarthritis and was associated with broken screws. CT scan was used to confirm the diagnose in this case. However, patient was managed conservatively.
$1 / 3$ had symptomatic non-union with periosteal reaction and elevated CRP was treated by surgical intervention in a tertiary care hospital by another foot and ankle surgeon.

The functional outcome was evaluated in terms of pre and post-operative MOXFQ score (Figure 4).

Following surgery, the mean MOXFQ score was reduced from $(62.68 \pm 7.36)$ to $(21.43 \pm 12.87)$ for pre-op and post op calculations respectively with a $\mathrm{Cl}$ of $95 \%$ $p$ value $<0.0001$ and $t$ statistic of -28.16 calculated using student $t$ test. The secondary outcome involved the idea of overall satisfaction of patients following surgery (Figure 4). In our study the overall satisfaction rate was $89 \% .3 / 28$ patients were over all dissatisfied with the results of surgery (11\%). 1/28 (3.57\%) were unsatisfied with the results secondary to malunion (dorsiflexion an-

\section{Results}
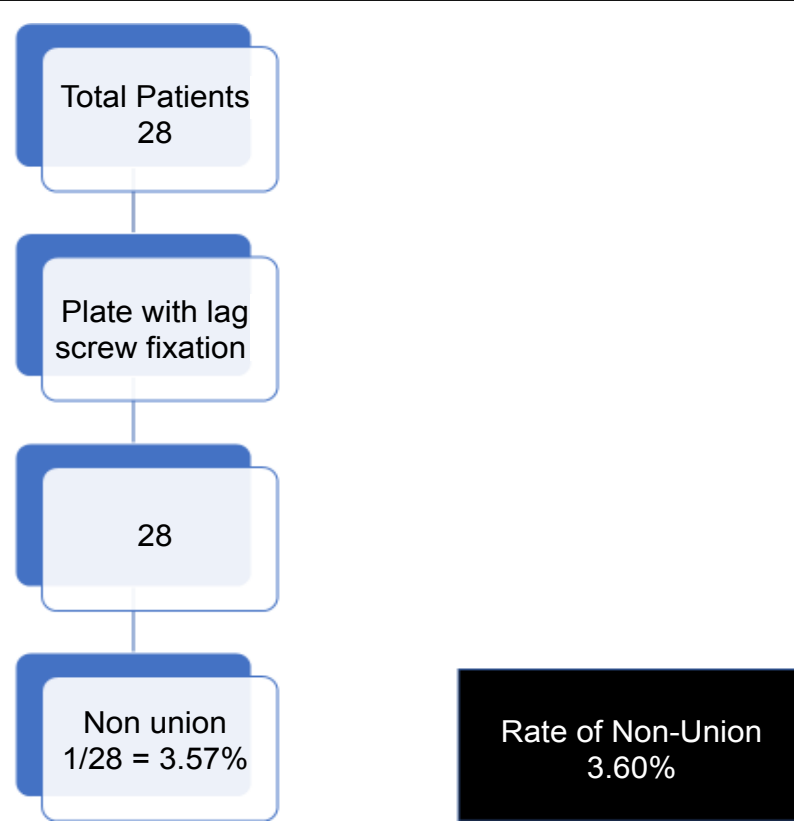

Figure 3: Patients suffered from non-union when plate with lag screw was used.

\section{Results}

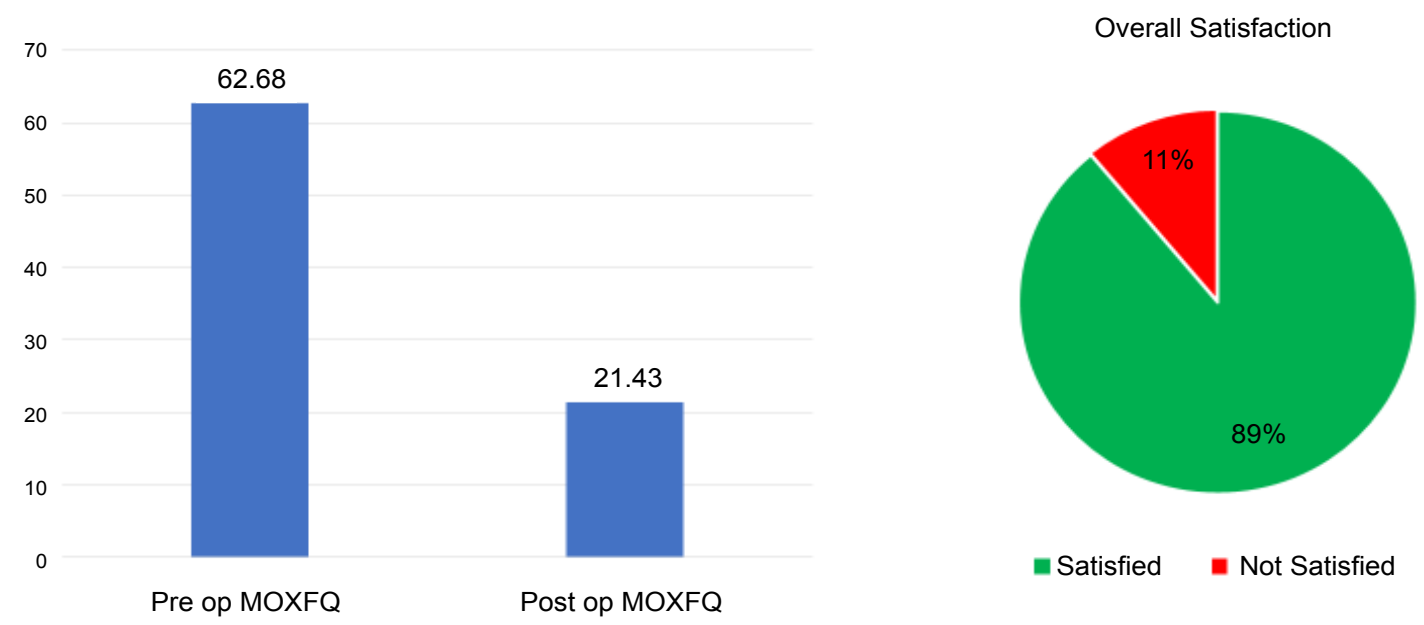

Figure 4: Overall satisfaction of patients following surgery. 
Table 1: Addition procedure along MTPJ fusion was performed in 7 out of 28 patients at the time of surgery.

\section{Additional Procedure:}

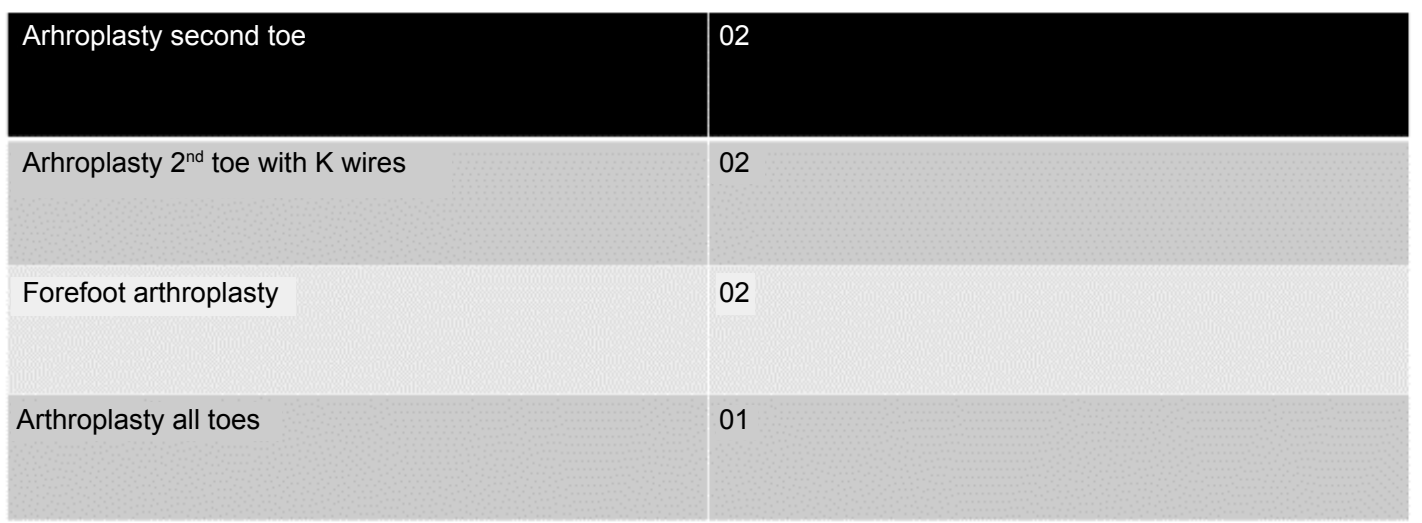

gle of 29 degrees) and was referred to other foot and ankle surgeon for further surgical intervention.

Addition procedure along MTPJ fusion was performed in 7 out of 28 patients at the time of surgery (Table 1).

\section{Discussion}

This is a retrospective single center study which evaluate the choice of implant for MTPJ fusion, functional outcome and patient satisfaction operated on a period of three years (2015-2018). We were able to evaluate locking plate with a lag screw.

The average age of our patients was 68.35 years which is comparable to what is reported in the literature [7]. There is predominance of female patient which in our case was $92 \%$ compared to what is reported in the literature i.e. $63 \%$ [8].

The choice of implant has been a matter of great debate for over the years when considering MTPJ fusion. The choice is dependent on the availability of implant, surgeon choice and experience. The dorsal plate with a lag screw offers increased stabilization in sagittal plan as reported by Significant lower mean dorsiflexion angle [8].

The use of cortical screw as part of per cutaneous technique has been documented with a fusion rate of $90 \%$. However, the results showed increasing non-union rate of $20 \%$ in case of elderly or patients with severe hallux valgus deformity [5].

This increased risk of non-union in patient with severe deformity supports the concept of most stable construct in case of patients with severe underlying disease like hallux valgus. Our experience (single center, single surgeon) with the cortical screw was extremely disappointing with a non-union rate of $2 / 3(33 \%)$, associated with hardware failure in $1 / 3$ cases. We proceeded with the use of locking plate with lag screw as an implant of choice in our center and evaluated the results as men- tioned above.

Cadaveric studies have demonstrated locking plate with lag screw was stiffer than non-locking plate with lag screw (considered as control during the study) with minimal displacement [2]. The idea of two locking plates at right angle without a compression screw has been associated with an unacceptable high non-union rate of $15 \%$ [6]. Our rate of non-union was $3.57 \%$.

The use of $\mathrm{K}$ wires with stimen pin introduced across interphalangeal joint was introduced by surgeons in one study as a simple and a cost-effective method. However, risk of interphalangeal joint arthritis has been documented as a possible risk [2].

Non-union rate was reported as highest in rheumatoid arthritis patient group i.e. $30 \%$ compared to osteoarthritis group i.e. $10 \%$ [6]. However, our study does not support this evidence, only one of our patients suffered from non-union and underlying etiology in this case was osteoarthritis. The non-union in this case was associated with hardware failure i.e. broken cortical screws. However, the non-union was asymptomatic and it was managed conservatively. In our set of patients, no one with rheumatoid arthritis suffered from non-union. In large trial of patients where various methods of fixation were employed patients suffering from hallux rigidus exhibited full rate of fusion compared to hallux valgus groups where $17 / 274$ (6.20\%) feet suffered from complication of non-union [7].

Our results demonstrated the non-union associated with hardware failure in $1 / 28$ patients $(3.60 \%)$, where we used plate with a lag screw as an implant of choice. However, in literature no failure of fusion rate was reported when they used locking plate as an implant of choice on 65 feet [7].

The post-operative course has also demonstrated a paradigm shift from orthodox approach of non-weight bearing to heel weight bearing. A systemic review of full weight bearing protocol after MTPJ fusion provides an 
acceptable rate of non-union of $6.35 \%$ [3]. Bearing this in mind we also allowed our patients full weight bearing immediately to avoid complications of non-weight bearing with acceptable non-union rate of $3.57 \%$.

\section{Conclusion}

We concluded that locking plate with lag screw is the implant of choice for MTPJ fusion of big toe with a nonunion rate of $3.60 \%$, good functional outcome, with significant reduction in pre and post-operative MOXFQ score.

\section{Limitation of Study}

It is a single center retrospective study with a small sample size. To improve the results and considering more weightage to use of plate with lag screw system effectiveness a higher number of patients might be required.

\section{References}

1. Daniel Mandell, John Karbassi, Hanbing Zhou, Brian Burroughs, Philip Aurigemma, et al. (2018) A locking compression plate versus the gold-standard non-locking plate with lag screw for first metatarsophalangeal fusion: A biomechanical comparison. Foot 34: 69-73.
2. Lawrence G Karlock, Levi Berry, Seth T Craft, Rocco Petrozzi, Adam G Grahn, et al. (2017) First metatarsophalangeal joint fusion with use of crossed kirschner wires and intramedullary steinmann pin. J Foot Ankle Surg 56: 11391142.

3. Kyle W Abben, Matthew D Sorensen, Brett J Waverly (2018) Immediate weight bearing after first metatarsophalangeal joint arthrodesis with screw and locking plate Fixation: A short-term review. J Foot Ankle Surg 57: 771-775.

4. Amanda Crowell, Jennifer C Van, Andrew J Meyr (2018) Early weight-bearing after arthrodesis of the first metatarsal-phalangeal joint: A systematic review of the incidence of non-union. J Foot Ankle Surg 57: 1200-1203.

5. Thomas Bauer (2017) Percutaneous first metatarsophalangeal joint fusion. Open Orthop J 11: 724-731.

6. Muhammad Ali Fazal, Jason Hol-Ming Wong, Luthfur Rahman (2018) First metatarsophalangeal joint arthrodesis with two orthogonal two-hole plates. Acta Orthop Traumatol Turc 52: 363-366.

7. Farshid Maleki, Ashwanth Ramesh, Adrian J Cassar-Gheiti, Ciara Fox, Paula Kelly, et al. (2019) Comparison of 4 different techniques in first metatarsophalangeal joint arthrodesis. Ir J Med Sci 188: 885-891.

8. Brent Cone, Jackson R Staggers, Sameer Naranje, Parke Hudson, Joseph Ingram, et al. (2018) First metatarsophalangeal joint arthrodesis: Does the addition of a lag screw to a dorsal locking plate influence union rate and/or final alignment after fusion. J Foot Ankle Surg 57: 259-263. 